\title{
Mechanism and Kinetics of Stabilization Reactions of Polyacrylonitrile and Related Copolymers III. \\ Comparison among the Various Types of Copolymers as Viewed from Isothermal DSC Thermograms and FT-IR Spectral Changes
}

\author{
Hideto $\mathrm{KAKIDA}^{\dagger}$ and Kohji TASHIRO* \\ Central Technology Research Laboratories, Mitsubishi Rayon Co., Ltd., Otake, \\ Hiroshima 739-06, Japan \\ * Department of Macromolecular Science, Graduate School of Science, Osaka University, \\ Toyonaka, Osaka 560, Japan \\ (Received October 31, 1996)
}

\begin{abstract}
Relationship between thermal behavior and structural change was studied by an organized combination of isothermal DSC thermograms and FT-IR spectra so as to investigate the stabilization reactions of acrylonitrile/acrylamide (AN/AAM) copolymer in comparison with those of acrylonitrile/methacrylic acid (AN/MAA) copolymer and polyacrylonitrile (PAN). The isothermal DSC exothermic thermogram of the AN/AAM copolymer was broader than that of the AN/ MAA copolymer and a little sharper than that of PAN, indicating that structural changes during stabilization proceed in the AN/ AAM sample more slowly than that in the AN/MAA copolymer and more rapidly than PAN homopolymer.

KEY WORDS Polyacrylonitrile / Acrylonitrile-Acrylamide Copolymer / Acrylonitrile-Methacrylic Acid Copolymer / Carbon Fiber / Stabilization / DSC / FT-IR /
\end{abstract}

Polyacrylonitriles (PAN) containing acidic comonomers are the most commonly used precursors for carbon fibers because of the excellent performance of PAN-based carbon fibers. ${ }^{1}$

In the process of PAN based-carbon fibers, stabilization is most important, because the physical properties of carbon fibers are affected significantly by the conditions of this process. The acrylic fibers are converted into infusible and nonflammable fibers by heating at $200-300^{\circ} \mathrm{C}$ for about one hour in an oxidative atmosphere during stabilization process. That is to say, the stabilization process proceeds quite slowly in a time scale of hours, largely different from the carbonization or pre-carbonization process occurring in several minutes. Only the thus stabilized fibers can be heated up to the carbonization temperature $\left(1000-2000^{\circ} \mathrm{C}\right)$ in an inert atmosphere. $^{2}$

The acrylic fiber made of PAN homopolymer has not been used as carbon fiber precursor, because the stabilization of PAN homopolymer fibers requires very long time and the physical properties of the thus prepared carbon fibers are said to be not so good. To accelerate the stabilization reaction and improve the physical properties of carbon fibers, several comonomers such as methacrylic acid (MAA), acrylic acid (AA), acrylamide (AAM), or methacrylate (MA) are often copolymerized into PAN. In spite of a number of studies, the roles of the comonomers on the kinetics of the stabilization reaction and the mechanism of the stabilization reaction have not been so clear yet now. ${ }^{2}$

The history of the study for the stabilization reaction was summarized by Bashir. ${ }^{3}$

In order to clarify the kinetics and the/mechanism of the stabilization of PAN and related copolymers, we developed a new idea to combine the experimental data of isothermal DSC thermograms and FT-IR spectra. ${ }^{4}$

In previous papers, ${ }^{4,5}$ we found the relationship be-

\footnotetext{
${ }^{\dagger}$ To whom all correspondence should be addressed.
}

tween the thermal behavior and the structure changes through an organized combination of isothermal DSC thermograms and FT-IR spectra measured for the samples picked out during the stabilization reaction of an AN/MAA copolymer ${ }^{4}$ and PAN. ${ }^{5}$

The isothermal DSC thermograms of AN/AAM copolymer were found to show intermediate features between PAN and AN/MAA copolymer. The isothertmal DSC thermogram of the AN/AAM copolymer shows a sharp exothermic peak at early period and a small broad exothermic peak at later period.

The present study was made to clarify the difference of stabilization mechanism among the AN/AAM copolymer, PAN and the AN/MAA copolymer by applying our new method combining isothermal DSC thermograms and FT-IR spectra.

\section{EXPERIMENTAL}

\section{Materials}

PAN, AN/AAM (CP2), and AN/MAA (CP1) copolymers were obtained by aqueous suspension polymerization method with redox initiator. The compositions are listed in Table I. The weight-averaged molecular weights of these polymers were about $2 \times 10^{5} \mathrm{~g} \mathrm{~mol}^{-1}$. The polymer powders filtered through a 400 mesh screen were used for the DSC and FT-IR measurements.

\section{DSC Measurement}

The DSC measurements were carried out by using a

Table I. Compositions of specimens used in this study ${ }^{a}$

\begin{tabular}{ll}
\hline Polymer & Composition (wt. ratio in feed) \\
\hline PAN & Homopolymer \\
CP1 & AN/MAA $=98.5 / 1.5$ \\
CP2 & AN/AAM $=98 / 2$ \\
\hline
\end{tabular}

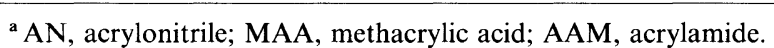


SEIKO DSC 220C controlled by a thermal analyzer SSC 5000 system. The sample weight of $4 \mathrm{mg}$ was used for the DSC measurements, because the rate of stabilization was the slowest for the $4 \mathrm{mg}$ weight samples among $1-10 \mathrm{mg}$ weight samples. The polymer powders were sealed in an $\mathrm{Al}$ pan with a mesh cover to allow air diffusion into powders. Isothermal DSC thermograms were measured at several constant temperatures between $170-260^{\circ} \mathrm{C}$.

\section{FT-IR Measurement}

IR measurements were made for the $\mathrm{KBr}$ disks (1 mg sample with $200 \mathrm{mg} \mathrm{KBr}$ ) for the specimens which were taken out of the DSC cell after heat treatment for predetermined periods. The JASCO FT-IR 7300 was used at a resolution of $4 \mathrm{~cm}^{-1}$ to detect the structural changes during the isothermal exothermic reactions observed in the DSC thermograms.

\section{RESULTS AND DISCUSSION}

\section{Isothermal DSC Measurements}

Figure 1 shows schematically the isothermal DSC thermograms of the CP2, the PAN and CP1 measured at $240^{\circ} \mathrm{C}$ under air flow. In Figure 1 , the thermograms of the CP2 and the PAN are about 20 and 100 times expanded longitudinally compared with that of the CP1. The thermogram of the CP1 shows apparently only one sharp exothermic peak in an early time region. The PAN exhibits the DSC thermogram with a small peak at an early period followed by a broad large peak at a later stage of reaction. The thermogram of the CP2 is intermediate between those of the CP1 and the PAN, showing a sharp exothermic peak at an early period and a broad small exothermic peak at later time region. In this way, the stabilization reactions of the $\mathrm{CP} 2$ are composed of rapid and slow exothermic processes as likely as that of the PAN, although the broad peak of the CP2 appears at the time period shorter than that of the PAN.

In Figure 2, the isothermal DSC thermogram and amount of heat evolved during stabilization processes are compared for $\mathrm{CP} 2$ and $\mathrm{CP} 1$. As indicated in a later section, the DSC thermograms are composed not only of the above-mentioned two (sharp and broad) peaks but also contain signals corresponding to the induction

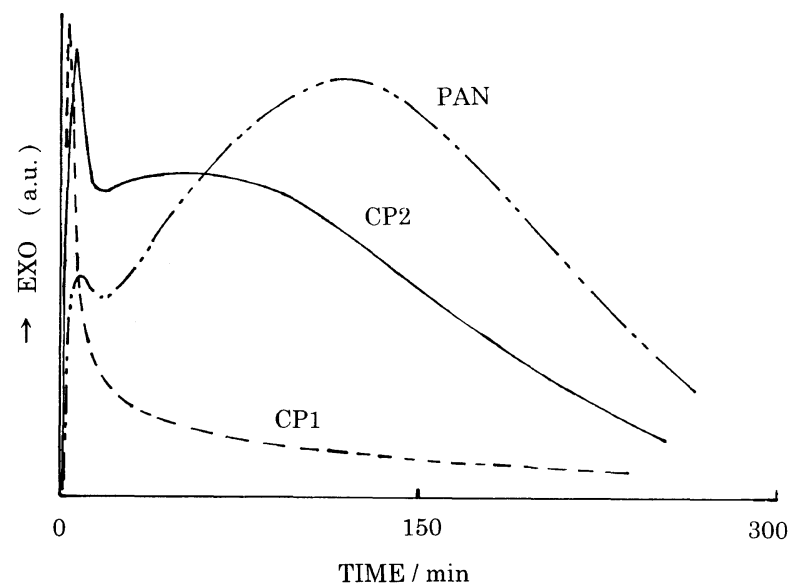

Figure 1. Isothermal DSC thermograms of PAN and of AN/AAM and AN/MAA copolymers measured at $240^{\circ} \mathrm{C}$ under air flow. period and shoulder observed in the earliest period of the thermograms (see Figure 3). The base lines necessary for evaluation of evolved heat were determined so that the heat flow level measured after enough long time of the reaction was equal to that in the induction period. The observation of the induction period in the stabilization reaction was made for the first time by us as described in detail in the previous report. ${ }^{4}$ The total amount of heat evolved during isothermal stabilization of the CP2 at $240^{\circ} \mathrm{C}$ is nearly the same as that of the CP1 and also close to that of the PAN homopolymer, ca. $3290 \mathrm{~J} \mathrm{~g}^{-1}$. ${ }^{5}$ The ordinate of Figure 2(a) is about 15 times that of Figure 2(b). So, it can be seen that the rate of heat evolution during the stabilization reaction of the CP2 is much slower than that of the CP1, but slightly faster than that of the PAN. From Figures 1 and 2, the comonomer AAM is considered to play a role accelerating the originally slow exothermic process of PAN
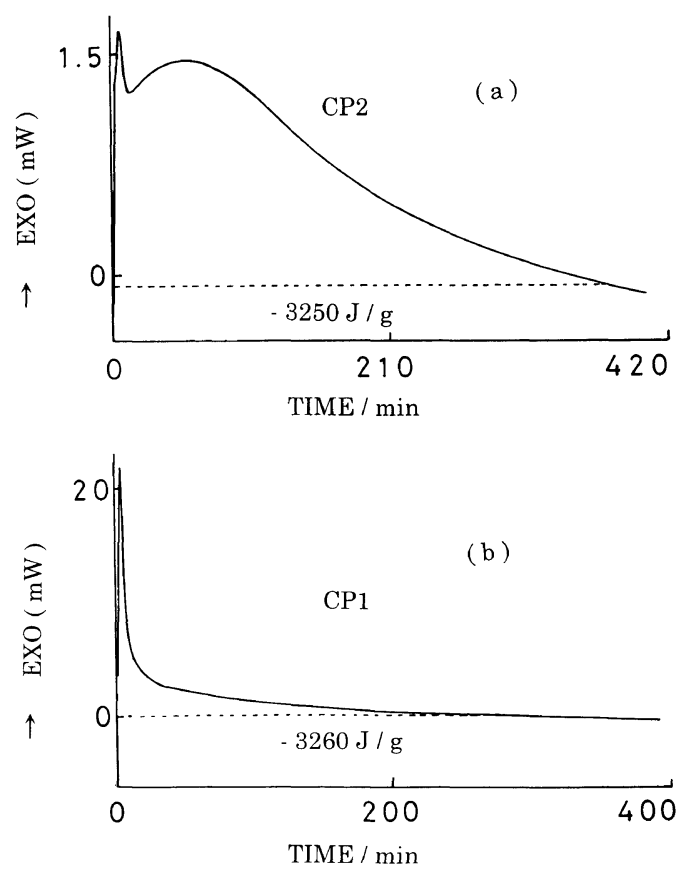

Figure 2. Isothermal DSC thermograms and total amount of heat evolved during stabilization at $240^{\circ} \mathrm{C}$ for (a) AN/AAM copolymer and (b) AN/MAA copolymer.

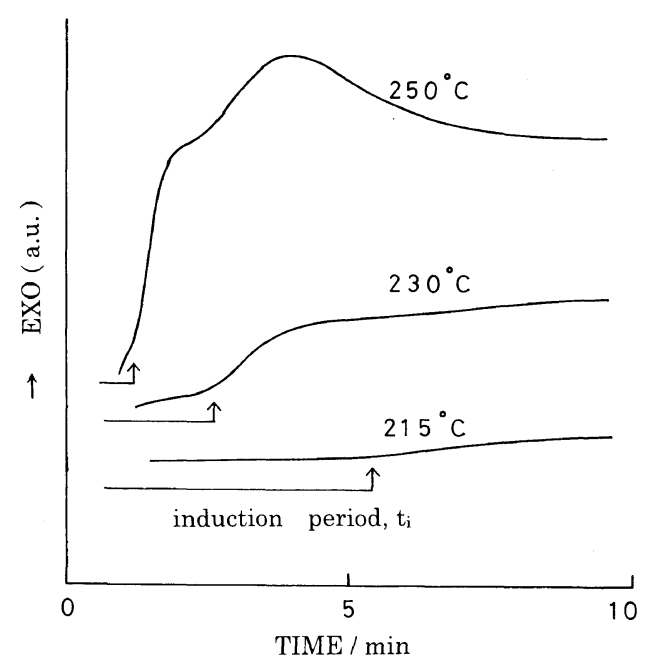

Figure 3. Isothermal DSC thermograms of AN/AAM copolymer measured at several temperatures under air flow. 
homopolymer but does not affect the total amount of heat evolution.

Figure 3 shows the isothermal DSC thermograms of the CP2 measured at various temperatures under air flow. In the early regions of these thermograms, there are nearly flat regions, the periods of which become shorter as the temperature is higher. These nearly flat regions should be considered as induction periods observed for the CP1 as reported in a previous paper. ${ }^{4}$ In Figure 4 , the Arrhenius plots are made for the induction periods obtained at $215,230,250$, and $260^{\circ} \mathrm{C}$, where the induction period $t_{\mathrm{i}}$ is defined as the standing point of the steep thermal evolution (see Figure 3). From the slope of the plot in Figure 4, activation energy for the initiation of the stabilization of the CP2 was evaluated as ca. $22 \mathrm{kcal} \mathrm{mol}^{-1}$. The corresponding values of the PAN and the CP1 were 22 and $26 \mathrm{kcal} \mathrm{mol}^{-1}$, respectively. ${ }^{4,5}$ The activation energies of these three polymers are almost the same, indicating that the initiation of stabilization reaction seems to be not accelerated by comonomers.

Structural Changes during Exothermic Reactions

Molecular structural changes of the CP2 during the

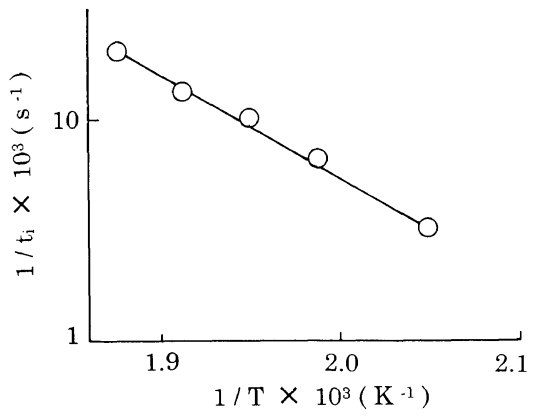

Figure 4. The Arrhenius plot of the induction period $\left(t_{\mathrm{i}}\right)$ measured at $215,230,250$, and $260^{\circ} \mathrm{C}$ for AN/AAM copolymer.

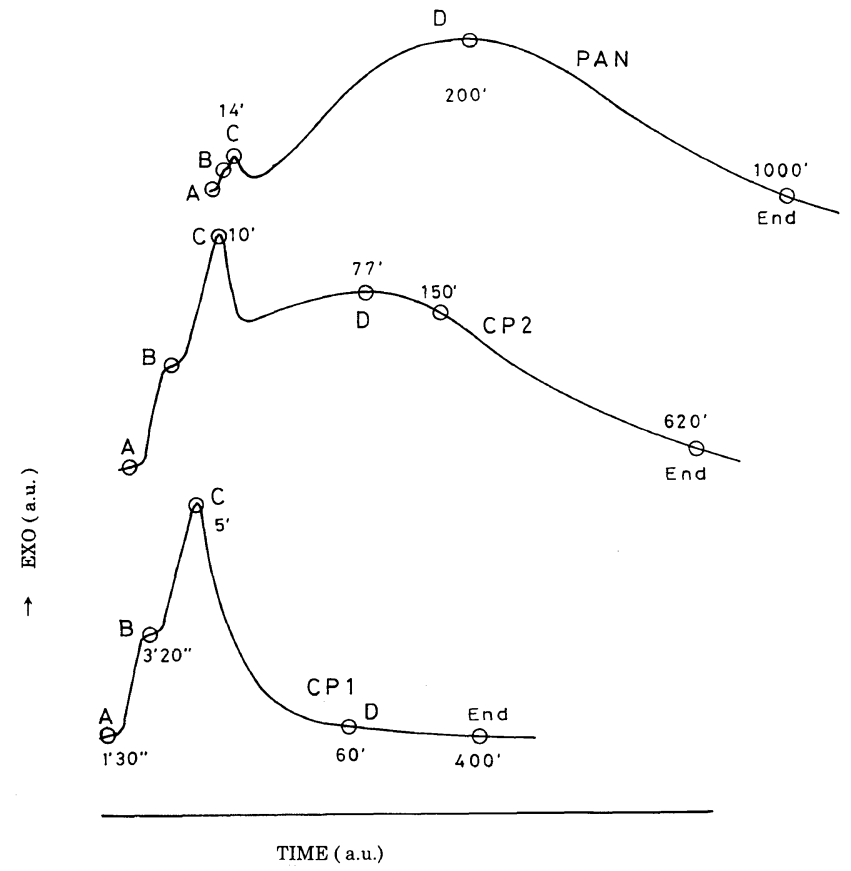

Figure 5. The schematic DSC thermograms of PAN and of $A N /$ AAM and AN/MAA copolymers measured at $230^{\circ} \mathrm{C}$ under air. The IR spectra were observed at the several points indicated by $O$, as shown in Figures 6, 7, and 8. isothermal heat evolution process were investigated by FT-IR. In Figure 5, a typical isothermal DSC thermogram of the CP2 measured at $230^{\circ} \mathrm{C}$ is illustrated schematically in comparison with those of PAN and CP1. The exothermic phenomena of the CP2 are apparently more complicated than that of the CP1 and rather resemble that of PAN. The thermogram of the CP2 consists of several components; (A) an induction period, (B) an exothermic shoulder, (C) a sharp peak and (D) a second broad peak as indicated in Figure 5. The broad peak of (D) is apparently absent in the thermogram of the CP1.

The IR spectra were measured at several points for the CP2 as indicated in Figure 5 to get information for the structural changes related with the isothermal exothermic DSC thermograms. The results were compared with the IR spectra of CP1 and PAN measured at several points as reported in the previous paper. ${ }^{4,5}$ The IR spectra are shown in Figures 6, 7, and 8 for CP2, CP1, and PAN, respectively. It can be seen that the bands at $2240,1450 \mathrm{~cm}^{-1}$ decrease in intensity while the bands at 1600 and $810 \mathrm{~cm}^{-1}$ increase in intensity as the annealing time in the DSC cell becomes longer. The assignments of the IR bands observed for the thermally degraded CP2, CP1, and PAN are shown in Table II with reference to the papers by Fochler et $a l^{6}$ and Sivy et $\mathrm{al}^{7}{ }^{7}$ By referring to the assignment in Table II, it is obvious that dehydrogenation of polymer backbone and nitrile oligomerization occur and as a result of these reactions the cyclic and aromatic structures are formed. When Figure 6 is compared with Figures 7 and 8 , the

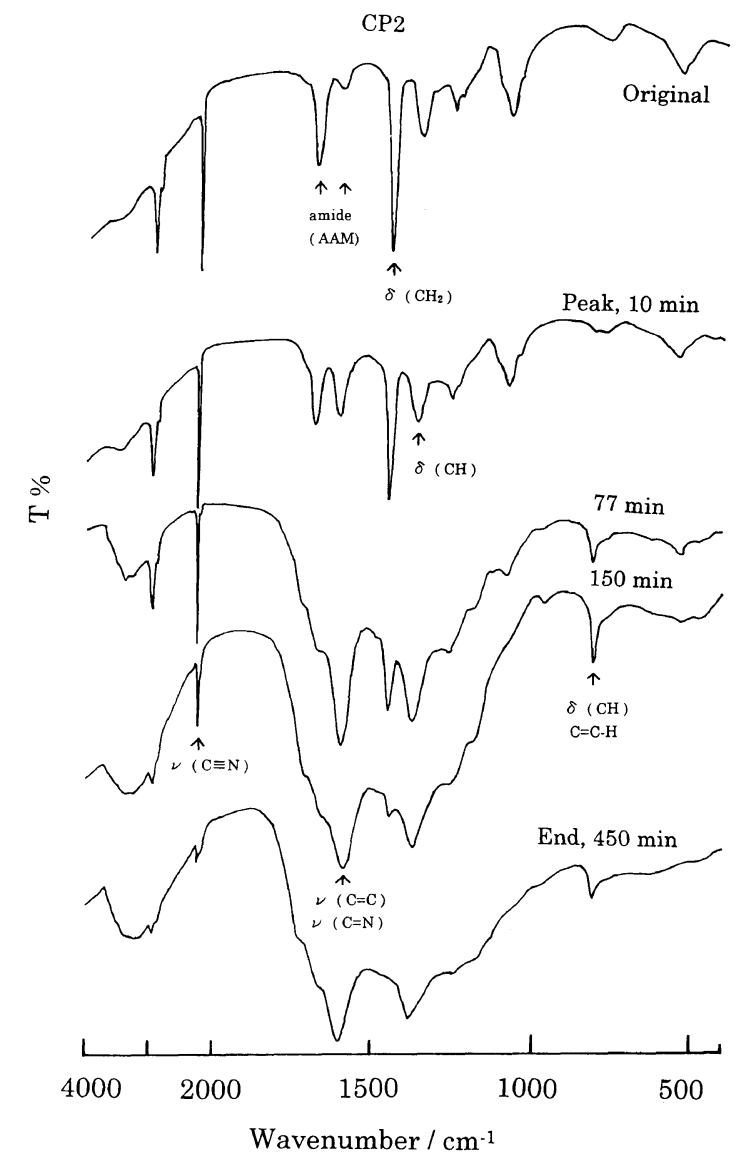

Figure 6. The IR spectra of AN/AAM copolymer which were observed at the points indicated by $\bigcirc$ shown in Figure 5 . 


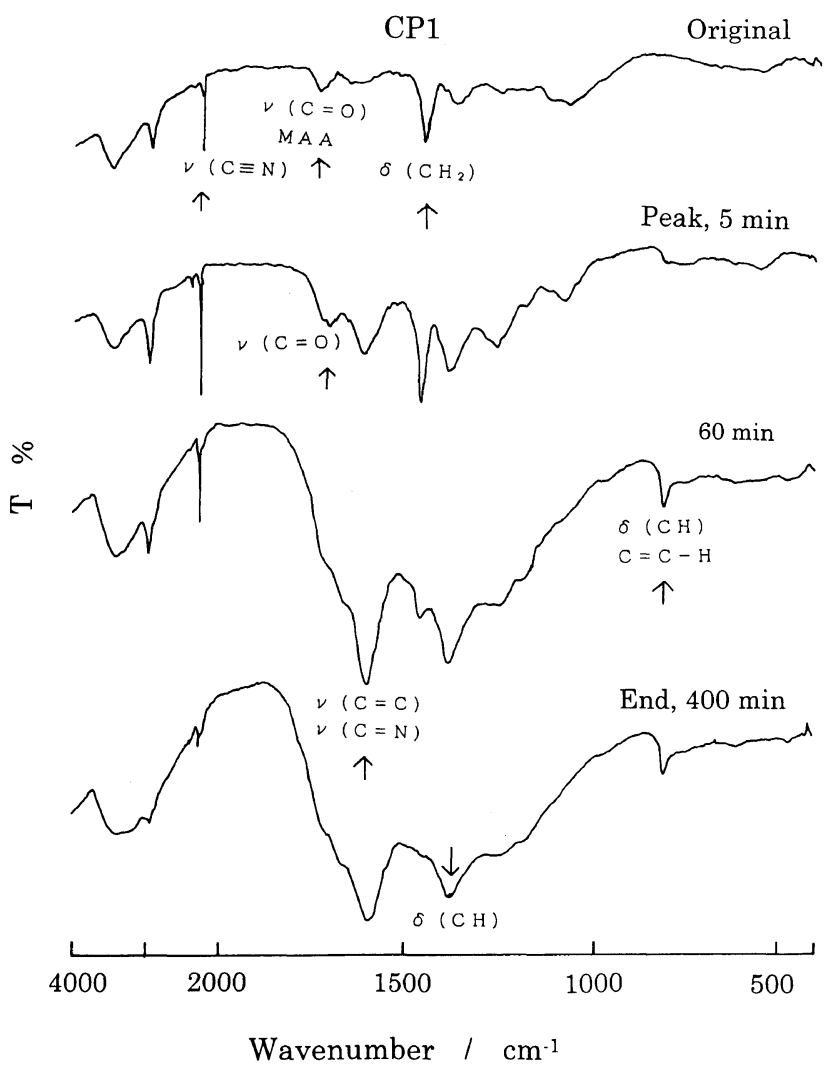

Figure 7. The IR spectra of AN/MAA copolymer which were observed at the points indicated by $O$ shown in Figure 5.

Table II. Principal IR bands in solid and degraded PAN, AN/MAA, and AN/AAM copolymer structures

\begin{tabular}{lc}
\multicolumn{1}{c}{ Functional groups } & Wavenumber $/ \mathrm{cm}^{-1}$ \\
\hline $\mathrm{NH}_{2}$ & 3390,3356 \\
$\mathrm{NH}$ & 3230 \\
$\mathrm{CH}_{2}$ & 2945,2920 \\
$\mathrm{CH}$ & 2895 \\
$\mathrm{C} \equiv \mathrm{N}$ (saturated) & 2240 \\
$\mathrm{C}=\mathrm{O}$ (unreacted MAA) & 1740 \\
$\mathrm{C}=\mathrm{O}$ (reacted MAA) & 1700 \\
$\mathrm{C}=\mathrm{O}$ (amide) & 1680 \\
$\mathrm{~N}-\mathrm{H}$ (amide) & 1620 \\
$\mathrm{C}=\mathrm{N}, \mathrm{C}=\mathrm{C}$ mixed & $1620^{\mathrm{a}}$ \\
$\mathrm{C}=\mathrm{N}, \mathrm{C}=\mathrm{C}, \mathrm{N}-\mathrm{H}$ mixed & $1580^{\mathrm{a}}$ \\
$\mathrm{CH}$ & 1450 \\
$\mathrm{CH}, \mathrm{NH}$ & 1380 \\
$\mathrm{C}-\mathrm{C}, \mathrm{C}-\mathrm{N}$ mixed & 1250 \\
$\mathrm{C}=\mathrm{C}-\mathrm{H}$ & 810
\end{tabular}

${ }^{a}$ These bands are often not resolved and appear at $1600 \pm 10 \mathrm{~cm}^{-1}$.

spectrum at the sharp peak position (C) of the CP2 is almost the same as those measured at the positions (C) of the CP1 and the PAN. The spectrum at broad peak position (D) of the CP2, contrary to the expectation, does not resemble that of the PAN, while the spectrum taken at $150 \mathrm{~min}$ position in the (D) region of the CP2 resembles the corresponding spectra of the PAN and the $\mathrm{CP} 1$. From these facts, it is assumed that the comonomers such as MAA and AAM accelerate preferably the large heat evolving reaction, i.e. dehydrogenation, than cyclization. The degree of acceleration of MAA is much larger than that of AAM.

In Figure 9, to get further information on differences

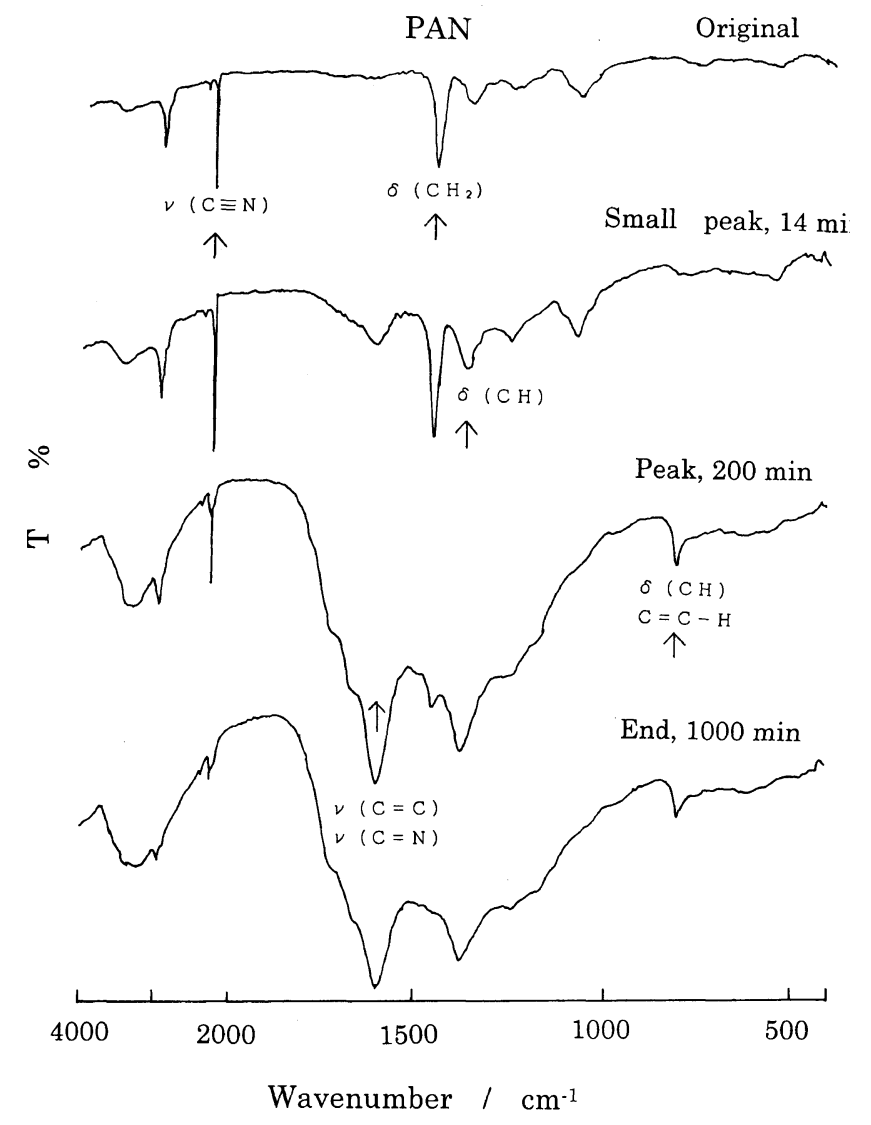

Figure 8. The IR spectra of PAN which were observed at the points indicated by $\bigcirc$ shown in Figure 5 .

of the structure changes between the CP2 and the CP1 during isothermal heat treatment under air, the isothermal DSC thermograms and changes of the several main IR band intensities are compared. As described in the previous paper ${ }^{4}$ in the case of the CP1, the IR band intensities change drastically in the exothermic region of early 50 min [regions $(A)-(C)$ ], the bands at 1600 and $810 \mathrm{~cm}^{-1}$ increase in intensity while the band at 2240 and $1450 \mathrm{~cm}^{-1}$ decrease. In the case of the $\mathrm{CP} 2$, the intensities of these IR bands change very slowly in an early period and only the $1450 \mathrm{~cm}^{-1}$ band changes in intensity somewhat more steeply than the other bands. One of the important phenomena detected in Figure 9 is that the $1740 \mathrm{~cm}^{-1}$ acid band of the CP1 decreases in intensity steeply while the $1680 \mathrm{~cm}^{-1}$ amide band of the CP2 decreases gradually, indicating that the AAM comonomer accelerates the stabilization reaction of the PAN, but the ability of acceleration is much smaller than the MAA comonomer, as already pointed out in the previous section.

To get information on the initiation reaction of stabilization of the CP2, structural changes during induction period were studied by FT-IR. In Figure 10, the changes of the IR spectra during the induction period caused by annealing in the DSC cell at $200^{\circ} \mathrm{C}$ under air flow are compared between $\mathrm{CP} 2$ and $\mathrm{CP} 1$. As described previous$1 y,{ }^{4}$ in the case of the CP1, the intensity of the $1740 \mathrm{~cm}^{-1}$ (MAA) band decreases and the $1700 \mathrm{~cm}^{-1}$ (reacted MAA) band appears and increases in intensity. This means that some MAA units react with the adjacent AN units in the induction period. For CP2, the amide 


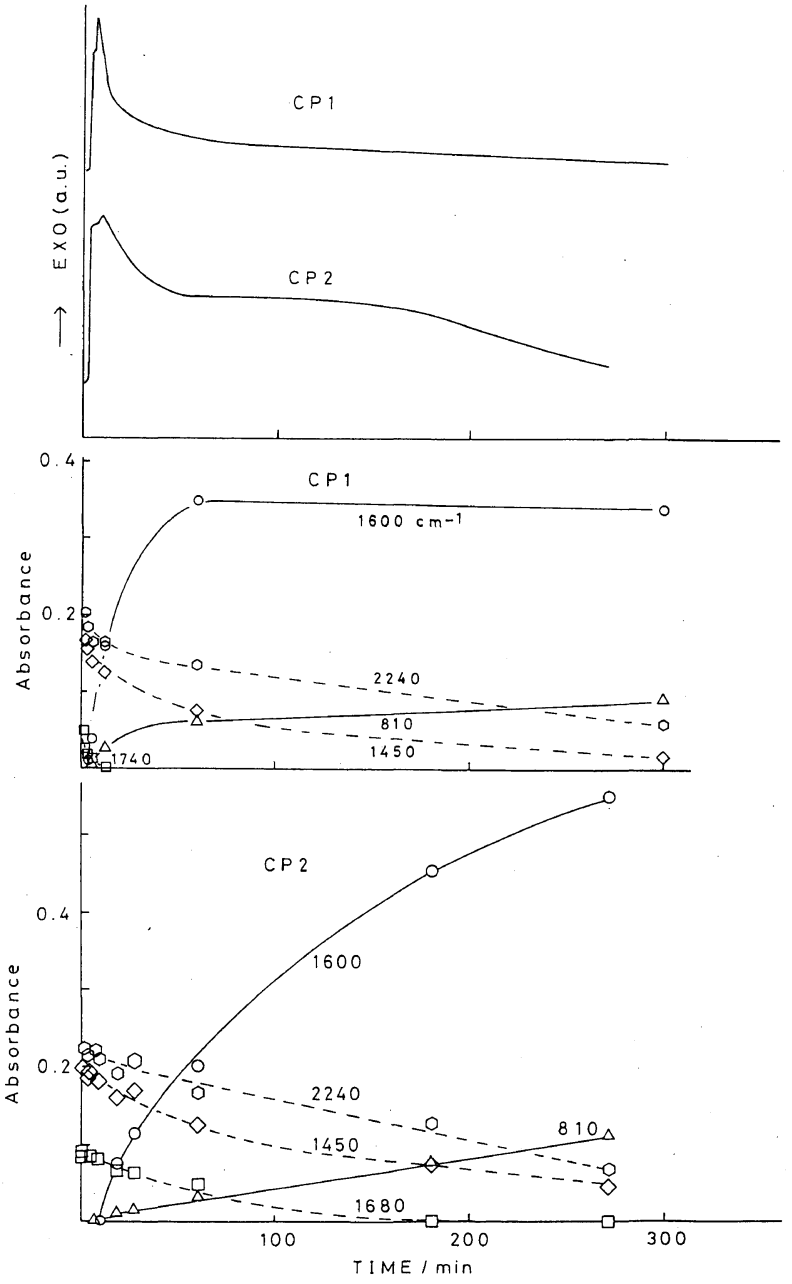

Figure 9. Comparisons of the isothermal DSC thermograms and the intensity changes of several main IR bands between AN/AAM and AN/MAA copolymers.

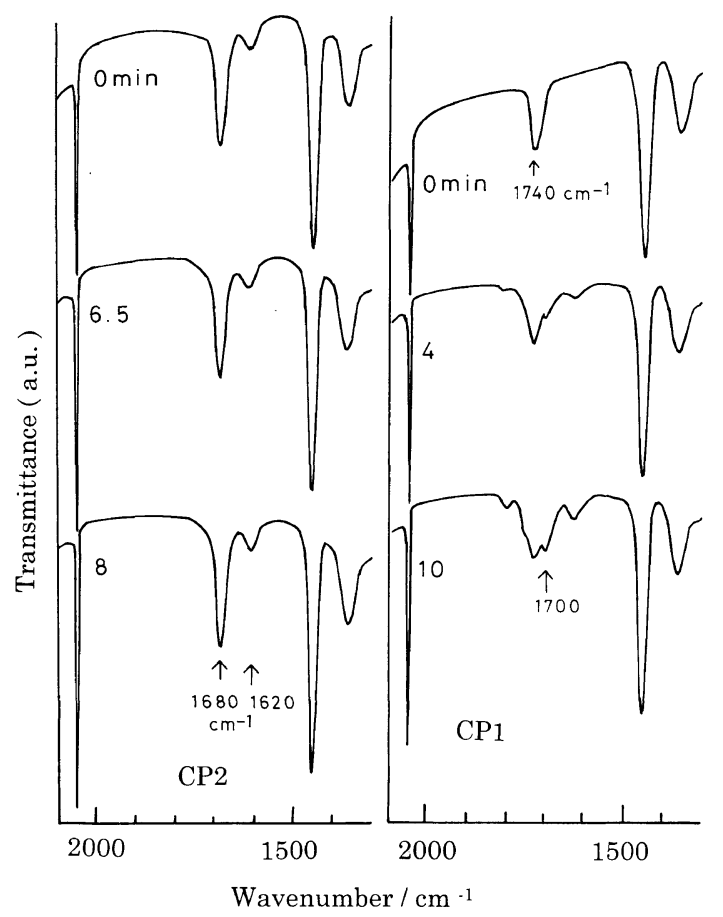

Figure 10. The changes of IR spectra during induction periods of AN/AAM and AN/MAA copolymers.
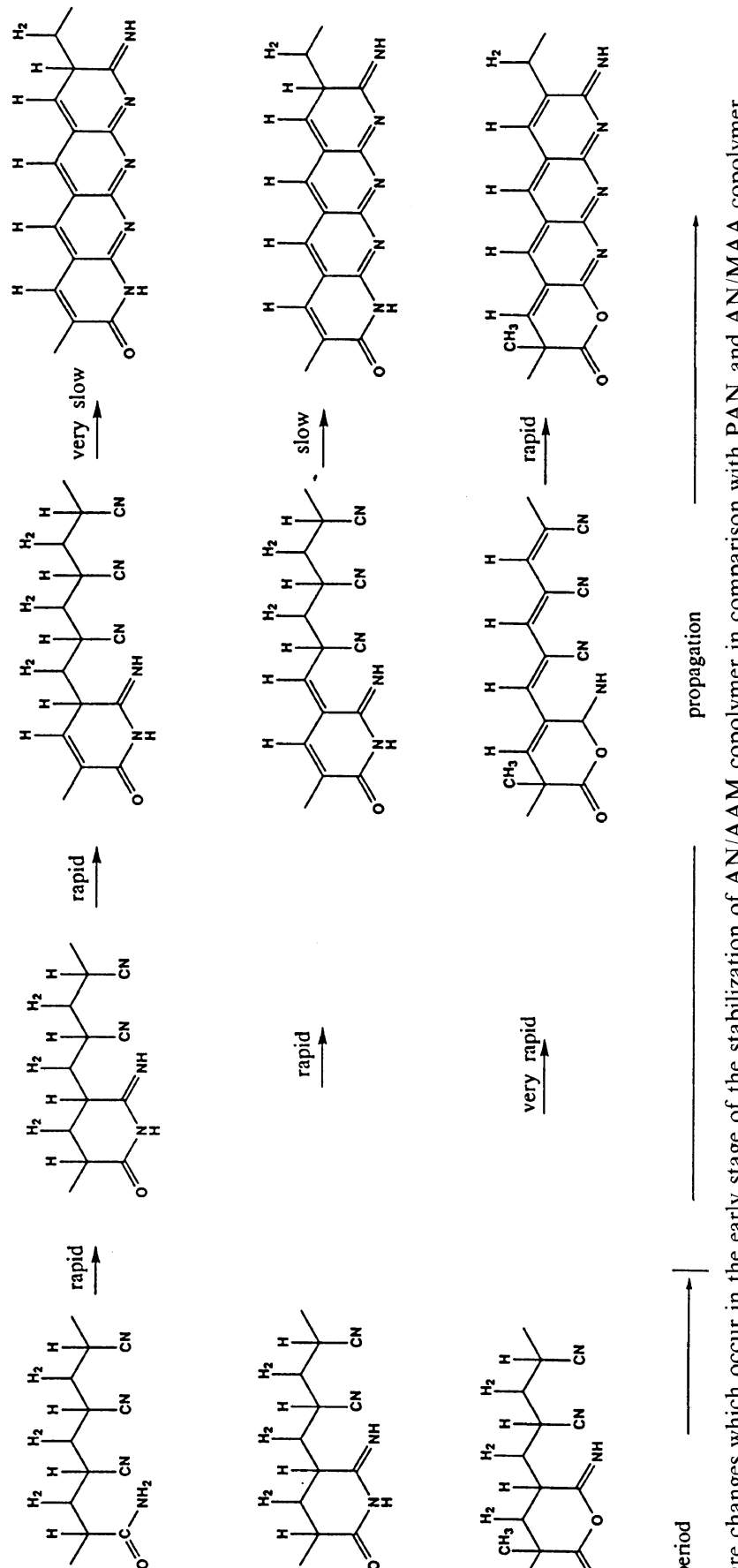

है।
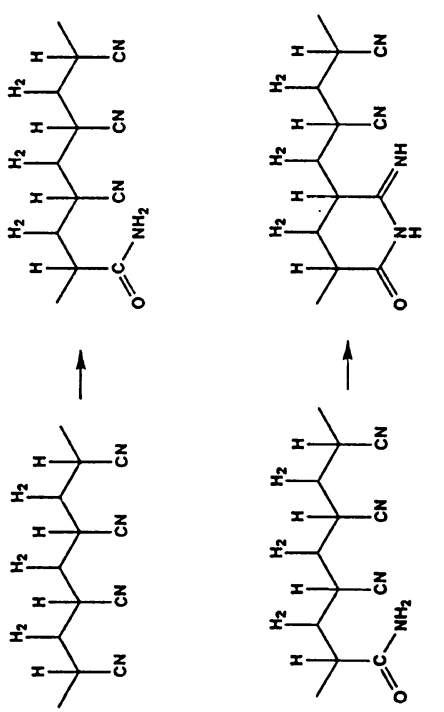

ב

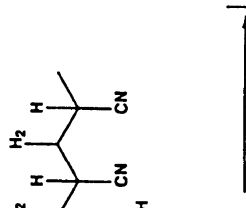

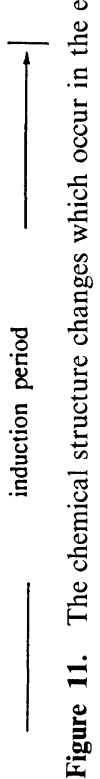

$\bar{\varepsilon}$ 
bands at 1620 and $1680 \mathrm{~cm}^{-1}$ show apparently no change in intensity. As described in the previous paper, ${ }^{5}$ the PAN homopolymer formed the amide groups from the nitrile groups during induction period. With reference to the PAN homopolymer, it may be considered that only small amounts of the amide groups react with adjacent nitrile groups in the case of the CP2. In other words, the CP2 is thermally more stable than the CP1 and more unstable than the PAN.

From the information obtained by the combination of isothermal DSC thermogram and FT-IR spectra and by referring to the IR band assignments in Table II, the stabilization mechanism of the CP2 may be described as illustrated in Figure 11.

At first, during the induction period, some amide groups of AAM units form the cyclic structures by coupling with the adjacent nitrile groups. And, when the amount of these cyclic structures reaches to a critical value, the propagation of the ring structures seems to occur. Following the induction period, in the heat evolution region (B) of Figure 5, the $1450 \mathrm{~cm}^{-1} \mathrm{CH}_{2}$ and $2240 \mathrm{~cm}^{-1} \mathrm{CN}$ bands decrease in intensity rather steeply. The $1450 \mathrm{~cm}^{-1}$ band decreases faster than the $2240 \mathrm{~cm}^{-1}$ band as shown in Figure 9. In this region, the dehydrogenation of the backbone should start through the reactions of amide groups of AAM with adjacent nitrile groups. In the exothermic sharp peak region $(\mathrm{C})$, the $1600 \mathrm{~cm}^{-1}$ band begins to appear and the $810 \mathrm{~cm}^{-1}$ band also appears slightly later in this region. After this exothermic sharp peak, the broad peak (D) is observed, where the 2240 and $1450 \mathrm{~cm}^{-1}$ band intensities decrease more slowly than in the sharp peak region $(\mathrm{C})$, and at the same time the 1600 and $810 \mathrm{~cm}^{-1}$ bands increase in intensity. From these experimental facts, we may say that the dehydrogenation of the backbone and the cyclization of nitrile groups proceed very slowly. After the broad peak, the pace of intensity change of the 2240,1450 , and $1600 \mathrm{~cm}^{-1}$ bands becomes slow, indicating that the dehydrogenation and cyclization reactions proceed very slowly in this region.

As obvious from Figure 9, in the case of CP1, the $1450 \mathrm{~cm}^{-1} \mathrm{CH}_{2}$ band intensity decreases more rapidly than that of the $2240 \mathrm{~cm}^{-1} \mathrm{CN}$ band during early $100 \mathrm{~min}$. After this period, the $\mathrm{CH}_{2}$ band decreases more slowly than the $\mathrm{CN}$ band. This indicates that, in the case of CP1, the large energy evolving dehydrogenation reaction mainly occurs in the early time region and the cyclization reaction proceeds rather slowly than the dehydrogenation. That is to say, MAA comonomer accelerates the dehydrogenation more than the cyclization reaction.

\section{CONCLUSIONS}

The present study compares the rates of the stabilization reaction of skeletal chains of PAN among the PAN homopolymer, the CP1 and the CP2 copolymers.

On the stabilization of the CP2, at first, some amide groups of AAM form cyclic structures with nitrile groups as illustrated in Figure 11, and these cyclic structures propagate to the larger structure through the nitrile oligomerization and dehydrogenation, and dehydrogenation occurs rather more predominantly than the cyclization. The amount of heat evolution of dehydrogenation is said to be $243 \mathrm{~kJ} \mathrm{~mol}^{-1}$ which is larger than the $59 \mathrm{~kJ} \mathrm{~mol}^{-1}$ of cyclization. ${ }^{1}$ That dehydrogenation is more predominant than the cyclization may be one reason why the sharper exothermic peak is observed for the CP2 case in the early time region than that of the PAN (Figure 1). On the stabilization of the CP1 which contains MAA as comonomer, the dehydrogenation and cyclization reactions proceed faster than those of the CP2. The $1740 \mathrm{~cm}^{-1}$ acid band of the CP1 decreases much more rapidly than the $1680 \mathrm{~cm}^{-1}$ amide band of the CP2. The dehydrogenation proceeds faster and slows down at more early stage than the cyclization. The comonomer MAA seems to accelerate the dehydrogenation and also the cyclization more largely than the comonomer AAM as shown in Figure 11. The acceleration of stabilization by such comonomers as MAA and AAM is more effective for the reaction of dehydrogenation than for cyclization.

\section{REFERENCES}

1. A. Gupta, D. K. Paliwal, and P. Bajaj, J. Macromol. Sci., C31, 1 (1991)

2. J. B. Donnet and R. C. Bansal, "Carbon Fiber," 2nd ed, Marcel Dekker, New York, N.Y., 1990.

3. Z. Bashir, Carbon, 29, 1081 (1991).

4. H. Kakida, K. Tashiro, and M. Kobayashi, Polym. J., 28, 30 (1996).

5. H. Kakida and K. Tashiro, Polym. J., submitted.

6. H. S. Fochler, J. R. Mooney, L. E. Ball, R. D. Boyer, and J. G. Grasseli, Spectrochim. Acta, 41A, 271 (1985).

7. G. T. Sivy and M. M. Coleman, Carbon, 19, 127 (1981). 